Rosália Maria de Oliveira ${ }^{1}$

Lúcia Helena Pinto Bastos 2

Ana Elisa Xavier de Oliveira e Dias 3

Sérgio Alves da Silva 1

Josino Costa Moreira 3

\section{Concentração residual de hexaclorociclohexano em área contaminada na Cidade dos Meninos, Duque de Caxias, Rio de Janeiro, Brasil, após tratamento com óxido de cálcio}

\author{
Residual concentration of hexachlorocyclohexane \\ in a contaminated site in Cidade dos Meninos, \\ Duque de Caxias, Rio de Janeiro, Brazil, after \\ calcium oxide treatment
}

\footnotetext{
1 Departamento de Saneamento e Saúde Ambiental, Escola Nacional de Saúde Pública, Fundação Oswaldo Cruz. Rua Leopoldo Bulhões 1480, Rio de Janeiro, $R J$ 21041-210, Brasil. rosalia@ensp.fiocruz.br sergio2000chem@yahoo.com

2 Instituto Nacional de Controle de Qualidade em Saúde, Fundação Oswaldo Cruz. Av. Brasil 4365, Rio de Janeiro, $R J$ 21045-900, Brasil. lucia@incqs.fiocruz.br 3 Centro de Estudos da Saúde do Trabalhador e Ecologia Humana, Escola Nacional de Saúde Pública, Fundação Oswaldo Cruz. Rua Leopoldo Bulhões 1480, Rio de Janeiro, $R J$ 21041-210, Brasil. aexodias@ensp.fiocruz.br josinocm@ensp.fiocruz.br
}

\begin{abstract}
Hexachlorocyclohexanes (HCHs) are currently considered potentially carcinogenic to humans. In Brasil, severe soil contamination by HCHs has been identified at Cidade dos Meninos, Duque de Caxias, Rio de Janeiro, where an old HCH factory was abandoned 40 years ago, leaving 1,700 local residents at risk. Calcium oxide treatment was performed on the most heavily contaminated (focus) area. Analysis of superficial soil samples collected years after treatment still showed high residual $\mathrm{HCH}$. Contamination levels as high as $6,200 \mathrm{mg} / \mathrm{Kg}$ and 7,320mg/Kg were found for $\alpha$ - and $\beta-H C H$ isomers. For the $\gamma$ - and $\delta$-HCH isomers, concentrations reached up to $140 \mathrm{mg} / \mathrm{Kg}$ and $530 \mathrm{mg} / \mathrm{Kg}$, respectively. The results showed that calcium oxide treatment was not efficient to decontaminate the soil in this area.

Key words Hexachlorocyclohexane; Contamination; Soil; Carcinogens; Decontamination

Resumo Os hexaclorociclohexanos (HCHs) são atualmente considerados carcinógenos humanos. No Brasil, uma severa contaminação de solo causada por HCHs é observada na Cidade dos Meninos, Duque de Caxias, Rio de Janeiro, onde uma antiga fábrica desses compostos foi abandonada há quarenta anos, ameaçando seus 1.700 habitantes. Para remediar a área pesadamente contaminada (área foco), um tratamento com óxido de cálcio foi realizado. Análises de solo coletadas superficialmente na área tratada, anos após o tratamento, mostraram ainda altas concentrações residuais de HCHs. Níveis de concentração tão alto quanto $6.200 \mathrm{mg} / \mathrm{Kg}$ e $7.320 \mathrm{mg} / \mathrm{Kg}$ foram encontrados para os isômeros $\alpha-e \beta-H C H$. Para os isômeros $\gamma-e \delta-H C H$, as concentrações observadas foram superiores a $140 \mathrm{mg} / \mathrm{Kg}$ e $530 \mathrm{mg} / \mathrm{Kg}$, respectivamente. Estes resultados mostraram que o tratamento com óxido de cálcio não foi eficiente para a descontaminação do solo desta área.

Palavras-chave Hexaclorociclohexano; Contaminação; Solo; Carcinógenos; Descontaminação
\end{abstract}




\section{Introdução}

A Cidade dos Meninos é uma área de 20 hectares localizada no Km 13 da Avenida Presidente Kennedy, no Município de Duque de Caxias, Rio de Janeiro. Nesta área foi construída em 1950 uma fábrica para sintetizar e formular o pesticida hexaclorociclohexano $(\mathrm{HCH})$, para ser usado no controle de vetores da doença de Chagas e da malária, entre outras (Bijos, 1961).

O produto era sintetizado por meio de adições sucessivas de cloro ao benzeno, gerando os isômeros $\alpha-, \beta-, \gamma-$, e $\delta$-HCH em diferentes percentuais estequiométricos. $\mathrm{O}$ isômero $\gamma$ $\mathrm{HCH}$, de maior ação inseticida, era sintetizado com um percentual de 14 a $15 \%$ da mistura técnica. A fábrica foi desativada em 1960 (Bastos, 1999). Os resíduos do HCH foram abandonados no local (Oliveira, 1994) e eram constituídos principalmente pelos isômeros $\alpha-, \beta-$ e $\delta-H C H$, formados na reação de síntese, não utilizados como inseticida.

Estes resíduos ficaram expostos in natura sobre o solo até 1989, junto com as ruínas da antiga fábrica, perfazendo uma área diretamente atingida, de cerca de $13.000 \mathrm{~m}^{2}$, situada no $\mathrm{Km}$ 2 da Estrada Camboaba, única via de acesso à Cidade dos Meninos (FEEMA, 1991).

Os isômeros do $\mathrm{HCH}$ podem contaminar não só o meio ambiente como também a população que tenha contato direto ou indireto com os resíduos. Esses são apolares, têm baixa solubilidade em água e alta estabilidade no meio ambiente, o que dificulta a degradação dos mesmos. De acordo com a Secretaria Nacional de Vigilância Sanitária, o HCH é classificado como Classe I, extremamente tóxico (SNVS, 1985, apud Mello, 1999). Segundo o Centro Internacional de Investigação sobre Câncer, o $\mathrm{HCH}$ é "possivelmente carcinogênico a humanos” (IARC, 2002). A contaminação humana pode ocorrer de forma aguda ou crônica, por meio das vias dérmica, respiratória e alimentar. Os isômeros do HCH são altamente lipofílicos e persistentes, sendo armazenados nos tecidos gordurosos. Assim, mesmo sendo absorvidos em pequenas quantidades, se a exposição é prolongada (crônica), efeitos adversos à saúde podem ser observados, como por exemplo, danos ao sistema nervoso central (WHO, 1991, 1992).

Em 1989, foi oficialmente constatada a contaminação ambiental da área da Cidade dos Meninos (FEEMA, 1991). Desde então, foi também detectada a presença dos isômeros do HCH no soro sangüíneo da população local. A contaminação na Cidade dos Meninos foi reconhecida como um problema de Saúde Públi- ca e Ambiental, e a partir de 1991, foram iniciados estudos para melhor caracterizar o problema e promover a descontaminação do local e o acompanhamento da saúde da população exposta (Braga, 1996; Oliveira, 1994).

Em 1995, houve tentativa de remediação da área foco, por uma empresa privada. A estratégia de tratamento adotada foi remediação química, in situ, por meio da adição de óxido de cálcio (cal) no solo contaminado (Dias et al., 1997). A área tratada com cal foi cercada perfazendo um total aproximado de $33.000 \mathrm{~m}^{2}$, duas vezes e meia o tamanho da área original estimada pela Fundação Estadual de Engenharia e do Meio Ambiente (FEEMA). A área foi considerada descontaminada, após noventa dias da utilização da cal, pelo executor do tratamento. Apenas o isômero $\gamma$-HCH foi monitorado para emissão do laudo de descontaminação (Bastos, 1999).

No entanto, mesmo após o referido tratamento, o odor de mofo, característico do $\mathrm{HCH}$, permanecia na área foco, indicando a ineficácia do processo. Além disso, considerando-se a estabilidade química do $\mathrm{HCH}$, tornava-se improvável que a reação química de degradação do $\mathrm{HCH}$ a triclorobenzenos (TCB), na presença de cal, ocorresse em condições ambientais brandas e sem controle reacional. Isto porque, em laboratório, a desalogenação desta classe de compostos requer condições extremamente enérgicas e controladas (Lin \& Hsieh, 1973).

Os resultados obtidos, indicam entre outros, que a metodologia utilizada não alcançou os objetivos propostos e a área continua contaminada com os quatro isômeros do $\mathrm{HCH}$. As concentrações residuais observadas atingiram a faixa de milhares de $\mathrm{mg} / \mathrm{Kg}$ para os isômeros $\alpha$ - e $\beta-\mathrm{HCH}$, e de centenas de mg/Kg para os isômeros $\gamma$ - e $\delta$ - $\mathrm{HCH}$.

O objetivo deste trabalho é, além de apresentar os resultados das análises de solo superficiais obtidas na área foco tratada, avaliar os níveis residuais de $\mathrm{HCH}$ após o tratamento com cal e, assim, determinar a sua eficácia.

\section{Metodologia}

\section{Coleta e preparo das amostras de solo}

Foram coletadas 44 amostras de solo superficial compostas da área foco tratada, em cada uma das direções cardeais, em pontos eqüidistantes com intervalo de $10 \mathrm{~m}$, até $100 \mathrm{~m}$ de distância em relação ao epicentro. Cada amostra foi composta por dez subamostras coletadas em um círculo imaginário de $2 \mathrm{~m}$ de diâmetro. 
O solo obtido foi acondicionado em folhas de papel Kraft recoberto com papel alumínio. Do total de amostras, 41 localizavam-se dentro da área foco tratada, e três outras nas proximidades de casas existentes até $200 \mathrm{~m}$ do epicentro da área foco tratada.

As amostras foram secas à temperatura ambiente, acondicionadas em frascos de vidro âmbar, previamente limpos, vedados com tampas de baquelite revestidas com filme de Teflon ${ }^{\circledR}$.

As amostras secas foram moídas, tamizadas (finalizando com malha de 20 mesh) e quarterizadas para completa homogeneização.

A partir de cada amostra homogeneizada foram obtidas três porções de $100 \mathrm{~g}$ cada. Estas foram acondicionadas em embalagens de alumínio e estocadas à temperatura de $-25^{\circ} \mathrm{C}$ para posterior análise.

A coleta das amostras de solo da área foco principal na Cidade dos Meninos, foi efetuada em dois dias consecutivos, 26 e 27 de setembro de 1997.

\section{Procedimento analítico}

Os isômeros do $\mathrm{HCH}$ foram extraídos da matriz de solo e purificados como descrito por Picó et al. (1994).

A cada $500 \mathrm{mg}$ de solo, adicionou-se $50 \mathrm{~mL}$ de mistura acetona: hexano 80:20 (v/v). A mistura de solo/solvente foi submetida à agitação por sessenta minutos, sonificada por $15 \mathrm{minu}$ tos e, posteriormente, filtrada utilizando-se filtro de papel Whatman 41 . O filtrado foi concentrado em rotavapor $\left(400 \mathrm{mmHg} / 45^{\circ} \mathrm{C}\right)$, a um volume final de $1 \mathrm{~mL}$. Os extratos concentrados foram purificados em colunas descartáveis de Florisil ${ }^{\circledR}$. Os compostos organoclorados foram, então, eluídos por meio da passagem de $50 \mathrm{~mL}$ da mistura de diclorometano: hexano 70:30 (v/v). A solução obtida foi novamente concentrada, até quase a secura, em rotavapor $\left(400 \mathrm{mmHg} / 45^{\circ} \mathrm{C}\right)$. O extrato obtido foi retomado em isooctano e concentrado sob atmosfera de nitrogênio super seco, a um volume final de $1 \mathrm{~mL}$.

\section{- Estabelecimento das condições cromatográficas}

As análises cromatográficas foram realizadas segundo a metodologia USEPA 608 (EPA, 1994). Soluções padrão de $\mathrm{HCH}$ foram preparadas em isooctano R. P. e utilizadas para o estabelecimento das condições cromatográficas.

A quantificação dos isômeros do $\mathrm{HCH}$, extraído das amostras de solo, foi feita por padronização externa.
As amostras de solo foram analisadas em duplicata, sendo utilizados os resultados médios das mesmas. A confirmação dos isômeros de $\mathrm{HCH}$, identificados por CG/DCE, foi realizada por meio de CG/EM.

As análises cromatográficas (GC/DCE) foram efetuadas em coluna capilar de sílica fundida nas seguintes condições:

a) Programação de temperatura do forno: $40{ }^{\circ} \mathrm{C}$ (2 min); $20^{\circ} \mathrm{C} / \mathrm{min} ; 180^{\circ} \mathrm{C}(10 \mathrm{~min}) ; 1,7{ }^{\circ} \mathrm{C} / \mathrm{min}$; $195^{\circ} \mathrm{C}$ ( $\left.2 \mathrm{~min}\right) ; 8^{\circ} \mathrm{C} / \mathrm{min}$; $280^{\circ} \mathrm{C}$ (15 min).

b) Temperatura do injetor: $280^{\circ} \mathrm{C}$.

c) Temperatura do detector: $300^{\circ} \mathrm{C}$.

d) Gás de arraste: hélio ultrapuro, a uma vazão de $3 \mathrm{~mL} / \mathrm{min}$.

e) Gás de (make up) do detector: mistura P5 (5\% de argônio em metano) com vazão de $30 \mathrm{~mL} / \mathrm{min}$.

f) Volume injetado: $1,0 \mu \mathrm{L}$.

g) Modo de injeção: com divisão de fluxo na razão de 1:10.

\section{Estudo da recuperação dos isômeros} do $\mathrm{HCH}$ da matriz de solo

Para o estudo de recuperação utilizou-se solo isento de contaminação por $\mathrm{HCH}$, com características físico-químicas semelhantes às do solo da Cidade dos Meninos, como descrito por Oliveira (1994). Este solo foi contaminado com soluções de $\mathrm{HCH}$ de concentrações conhecidas. O solo padrão foi submetido ao mesmo processo analítico descrito para as amostras de solo oriundas da Cidade dos Meninos.

\section{Materiais e reagentes}

- Cromatógrafo a gás Hewlett Packard (HP 5890 - Série II) com detector de captura de elétrons, com sistema de tratamento de dados;

- Rotavapor RE 120, Marca Buchi;

- Colunas descartáveis de Florisil (Bakerbond Spe*), fornecida por J. T. Baker Inc.;

- Coluna capilar de sílica fundida DB-5 (5\% de fenilmetilsiloxano, $30 \mathrm{~m} \times 0,32 \mathrm{~mm} \times 0,25 \mu \mathrm{m}$ ), fornecida por J. \&W. Scientific.

- Todas as substâncias de referência (isômeros do $\mathrm{HCH}$ ) foram fornecidas pela Agência Ambiental Americana (EPA).

- Todos os solventes utilizados foram grau resíduo de pesticida (R. P.) e fornecidos pela Omnisolv,

\section{Resultados}

A partir dos resultados obtidos, descritos na Tabela 1, pode-se observar que os isômeros $\alpha$ - 
Tabela 1

Concentrações de $\alpha-, \beta-, \gamma-$, e $\delta-H C H$, em solo da área foco da Cidade dos Meninos (mg/Kg),

após tratamento com cal. Duque de Caxias, Rio de Janeiro, Brasil.

\begin{tabular}{|c|c|c|c|c|c|c|c|c|c|c|c|}
\hline \multirow{2}{*}{$\begin{array}{l}\text { Concentração dos } \\
\text { isômeros nas direções } \\
\text { cardeais }(\mathrm{mg} / \mathrm{Kg})\end{array}$} & \multicolumn{11}{|c|}{ Distância do epicentro (m) } \\
\hline & 10 & 20 & 30 & 40 & 50 & 60 & 70 & 80 & 90 & 100 & 160 \\
\hline \multicolumn{12}{|l|}{$\alpha-\mathrm{HCH}$} \\
\hline$N$ & $1,6 \times 10^{3}$ & 41,8 & 29,0 & $5,1 \times 103$ & $1,4 \times 10^{3}$ & $6,2 \times 10^{3}$ & $5,7 \times 10^{3}$ & $1,9 \times 10^{3}$ & $2,0 \times 102$ & 2,1 & 7,8 \\
\hline $\mathrm{S}$ & 47,0 & 64,0 & $2,9 \times 103$ & $1,4 \times 10^{2}$ & NA & $1,4 \times 10^{2}$ & 15,0 & 3,4 & 8,1 & 4,8 & 1,7 \\
\hline$L$ & $1,5 \times 10^{2}$ & $5,6 \times 10^{2}$ & $2,7 \times 10^{2}$ & $1,2 \times 10^{3}$ & $1,3 \times 10^{3}$ & $1,1 \times 10^{3}$ & 3,6 & 1,3 & 4,4 & 4,6 & 1,4 \\
\hline $\mathrm{O}$ & $7,2 \times 10^{3}$ & $7,5 \times 10^{3}$ & $3,6 \times 10^{3}$ & $5,2 \times 10^{3}$ & $4,5 \times 10^{3}$ & $3,4 \times 10^{3}$ & $3,4 \times 10^{3}$ & 21,0 & 3,2 & 6,3 & NA \\
\hline \multicolumn{12}{|l|}{$\beta-\mathrm{HCH}$} \\
\hline$N$ & $2,2 \times 10^{3}$ & $9,9 \times 10^{3}$ & $8,5 \times 10^{3}$ & $5,2 \times 10^{3}$ & $12,4 \times 10^{3}$ & $65,3 \times 10^{3}$ & $10,1 \times 10^{3}$ & $26,7 \times 10^{3}$ & $1,6 \times 10^{3}$ & 15,3 & 50,6 \\
\hline $\mathrm{S}$ & 40,2 & $4,7 \times 10^{3}$ & $3,7 \times 10^{3}$ & $1,3 \times 10^{3}$ & NA & $1,1 \times 10^{3}$ & $1,4 \times 10^{2}$ & 50,5 & $1,2 \times 10^{2}$ & 84,1 & 16,2 \\
\hline$L$ & 7,5 & $3,7 \times 10^{3}$ & $1,1 \times 10^{3}$ & $24 \times 10^{3}$ & $4,2 \times 10^{3}$ & $5,9 \times 10^{3}$ & 38,5 & 17,4 & 39,6 & 53,2 & 6,7 \\
\hline $\mathrm{O}$ & $2,8 \times 10^{3}$ & $13,3 \times 10^{3}$ & $31,5 \times 10^{3}$ & $2,3 \times 10^{2}$ & $73,2 \times 10^{3}$ & $62,6 \times 10^{3}$ & $63,7 \times 10^{3}$ & ND & $1,0 \times 10^{3}$ & $1,1 \times 10^{2}$ & 34,9 \\
\hline \multicolumn{12}{|l|}{$\gamma-\mathrm{HCH}$} \\
\hline $\mathrm{N}$ & $1,4 \times 10^{2}$ & $3,8 \times 10^{2}$ & $1,1 \times 10^{2}$ & $4,0 \times 10^{2}$ & 56,2 & $7,2 \times 10^{2}$ & ND & $1,7 \times 10^{2}$ & 14,3 & ND & NA \\
\hline $\mathrm{S}$ & ND & ND & ND & 1,6 & NA & 6,2 & ND & 0,1 & ND & 0,1 & NA \\
\hline $\mathrm{L}$ & 8,7 & 8,4 & 14,0 & ND & 90,6 & 8,8 & ND & ND & ND & 0,8 & NA \\
\hline $\mathrm{O}$ & $5,8 \times 10^{2}$ & $1,4 \times 10^{3}$ & $1,1 \times 10^{2}$ & ND & ND & 6,7 & $1,7 \times 10^{2}$ & ND & ND & ND & NA \\
\hline \multicolumn{12}{|l|}{$\delta-\mathrm{HCH}$} \\
\hline $\mathrm{N}$ & 99,2 & $1,3 \times 10^{2}$ & 59,1 & $1,7 \times 10^{2}$ & 21,8 & $1,8 \times 10^{2}$ & 30,1 & $1,7 \times 10^{2}$ & 7,4 & 0,1 & 0,6 \\
\hline $\mathrm{S}$ & 7,4 & 3,4 & 3,4 & ND & NA & 1,3 & ND & 0,4 & 0,6 & ND & ND \\
\hline $\mathrm{L}$ & 3,1 & 43 & 8,1 & 5,3 & 64 & 5,7 & ND & 0,1 & ND & 0,1 & 0,1 \\
\hline $\mathrm{O}$ & 57,0 & $5,3 \times 102$ & $1,5 \times 102$ & 25,0 & $1,0 \times 102$ & $1,1 \times 102$ & 97,0 & 6,0 & ND & ND & ND \\
\hline
\end{tabular}

ND = Não detectado; NA = Não analisado

Limite de detecção do método: $1 \mu \mathrm{g} / \mathrm{Kg}$.

Os resultados expressos nesta tabela representam a média aritmética de duas análises, em mg de HCH por Kg de solo contaminado, amostrado nas direções cardeais, em distâncias crescentes $(\mathrm{m})$ em relação ao epicentro da área foco tratada com cal.

Período da coleta de amostras de solo: 26 e 27 de setembro de 1997.

$\mathrm{HCH}, \beta-\mathrm{HCH}, \gamma-\mathrm{HCH}$ e $\delta$ - $\mathrm{HCH}$ foram detectados nas amostras de solo oriundas da área foco após adição de cal, em concentrações da ordem $\mathrm{de} \mathrm{mg} / \mathrm{Kg}(\mathrm{ppm})$.

Para os isômeros $\alpha-\mathrm{HCH}$ e $\beta-\mathrm{HCH}$, os teores residuais observados atingiram a faixa de milhares de $\mathrm{mg} / \mathrm{Kg}$. As concentrações variaram, respectivamente, de 1,3 a $6.200 \mathrm{mg} / \mathrm{Kg}$ de solo e de não detectado (ND) a $7.320 \mathrm{mg} / \mathrm{Kg}$ de solo.

Para os isômeros $\gamma-\mathrm{HCH}$ e $\delta-\mathrm{HCH}$, os teores residuais observados foram mais baixos. No entanto, ainda assim, atingiram a faixa de centenas de mg/Kg. As concentrações variaram, respectivamente, de não detectado (ND) a 140mg/ Kg de solo e de não detectado (ND) a 530mg/Kg de solo.

As amostras obtidas nas direções Norte e Oeste foram as que apresentaram as concentrações mais elevadas, para todos os isômeros de $\mathrm{HCH}$ analisados.
Concentrações residuais elevadas, da ordem de $\mathrm{mg} / \mathrm{Kg}$ (ppm), foram observadas para os isômeros $\alpha-, \beta-$ e $\delta-\mathrm{HCH}$, também nas três amostras obtidas em quintais de residências (fora da área foco tratada, a 160m do epicentro). Os valores residuais máximos encontrados para os isômeros $\alpha$-, $\beta$ - e $\delta$-HCH foram, respectivamente, $7,8 \mathrm{mg} / \mathrm{Kg}$ de solo, $50,6 \mathrm{mg} / \mathrm{Kg}$ de solo e $0,6 \mathrm{mg} / \mathrm{Kg}$ de solo. O isômero $\gamma$-HCH não foi analisado nessa distância.

\section{Discussão}

Dentre os quatro isômeros analisados, o $\beta-\mathrm{HCH}$ é o que se encontra em maior concentração residual em todas as direções de amostragem $(\mathrm{N}$, $\mathrm{S}, \mathrm{L}, \mathrm{O}$ ). O isômero $\delta$ - $\mathrm{HCH}$ apresentou concentração aparentemente mais elevada do que o $\gamma$ $\mathrm{HCH}$. Isso se deve ao fato de uma das amostras 
ter apresentado concentração de $530 \mathrm{mg} / \mathrm{Kg}$ de solo para o isômero $\delta$-HCH. Na maior parte das amostras, no entanto, os isômeros encontravam-se na seguinte ordem de concentração: $\beta$ $\mathrm{HCH}>\alpha-\mathrm{HCH}>\gamma-\mathrm{HCH}>\delta-\mathrm{HCH}$, dados que confirmam os descritos por Oliveira (1994).

Concentrações residuais mais elevadas para o isômero $\beta-\mathrm{HCH}$ eram esperadas, já que este isômero é o que apresenta maior estabilidade devido à sua simetria. Portanto, pode permanecer por períodos mais prolongados no meio ambiente. Além disso, através de interconversão, o isômero $\alpha-\mathrm{HCH}$ pode formar $\beta-\mathrm{HCH}$ (Edwards, 1975).

Concentrações residuais elevadas para o isômero $\alpha-\mathrm{HCH}$ também eram esperadas, uma vez que este era o que se encontrava em maior percentual na mistura técnica produzida na Cidade dos Meninos (70 \%).

$\mathrm{O}$ isômero $\gamma-\mathrm{HCH}$ era produzido em percentual estequiométrico menor que os de $\alpha-\mathrm{HCH}$ e $\beta-\mathrm{HCH}$, separado da mistura técnica e comercializado como inseticida. Portanto, eram esperadas concentrações inferiores em relação aos isômeros $\alpha$-HCH e $\beta$-HCH.

$\mathrm{O}$ isômero $\delta$ - $\mathrm{HCH}$ era o produzido em menor percentual estequiométrico. Assim, sua concentração, como contaminante no solo, deveria ser menor que a dos demais. Os resultados aqui apresentados estão em acordo com o esperado.

No presente trabalho, pode-se observar que as concentrações residuais do isômero $\gamma-\mathrm{HCH}$ encontradas nas matrizes de solo (até 140mg/ $\mathrm{Kg}$ ), após a adição de cal, foram superiores às descritas pela empresa executora do referido tratamento.

A empresa, embora não tenha citado as concentrações iniciais de $\gamma-\mathrm{HCH}$ (único isômero monitorado por esta) no solo antes do tratamento, garantiu um percentual médio de redução do isômero $\gamma$-HCH nas quatro amostras analisadas, após o tratamento realizado, em 99,18\% (Nortox S.A., 1995, apud Bastos, 1999). Se este percentual de redução tivesse realmente sido atingido, o esperado seria encontrar concentrações de $\gamma$-HCH da ordem de no máximo unidades de mg/Kg, já que os valores encontrados por Oliveira (1994), na mesma área, anteriormente ao tratamento, tinha valores máximos de $\gamma$-HCH de $191 \mathrm{mg} / \mathrm{Kg}$.

Parece óbvio que a avaliação da eficiência de um processo de remediação deve ter como ponto de partida o conhecimento das concentrações iniciais de todas as espécies de interesse presentes no meio e não apenas de uma delas, que não é inclusive, a mais estável ou abundante.
No entanto, a concentração de $\gamma$-HCH observada nas amostras analisadas no presente trabalho, foi de até $140 \mathrm{mg} / \mathrm{Kg}$, ou seja, da mesma ordem de grandeza descrita por Oliveira (1994). Isso confirma a ineficácia do tratamento, já que as concentrações de $\gamma$-HCH permanecem inalteradas.

Se o $\gamma$-HCH é o isômero, dentre os quatro estudados, que apresentava menor estabilidade, e este não havia sido degradado, era de se esperar que os outros três permanecessem como contaminantes após o tratamento com cal. Os resultados apresentados, então, estão de acordo com o esperado.

A presença de $\mathrm{HCH}$ em concentrações elevadas, nas três amostras obtidas nos quintais de residências, indicam contaminação do solo mesmo fora da área tratada. Isso pode ser explicado pelo fato dos resíduos de $\mathrm{HCH}$, da antiga fábrica, serem utilizados nas hortas de várias residências para combate a pragas. Estoques de HCH são encontrados em algumas residências. Assim, esta contaminação observada pode ser devida ao transporte mecânico de $\mathrm{HCH}$, a partir da área foco, por parte dos residentes.

Além do transporte mecânico, nas residências mais próximas à área tratada (de onde foram obtidas as amostras de solo aqui analisadas), o HCH pode também ter sido transportado, a partir do foco, através de lixiviação em períodos chuvosos. Nesses períodos, a área foco, situada em cota ligeiramente superior à das residências, é inundada devido às características topográficas e geológicas da região, facilitando o seu carreamento pelas águas (Oliveira, 1994).

A contaminação de outras áreas na Cidade dos Meninos por $\mathrm{HCH}$, também já havia sido observada por Oliveira (1994). Essa contaminação, tanto ambiental como da população local (Braga, 1996), indicavam uma dispersão dos contaminantes na área como um todo. Quanto à contaminação da população, Braga sugeriu que esta poderia ter ocorrido por meio de vias dérmica, respiratória e por intermédio do consumo de alimentos cultivados na área. A detecção de $\mathrm{HCH}$ (no presente trabalho) nas amostras de solo dos quintais, onde são cultivados alimentos de subsistência, corroboram a hipótese de contaminação via alimentar, apontada por Braga (1996).

\section{Conclusão}

Segundo o presente trabalho, pode-se concluir que a ordem de grandeza (até milhares de mg/ $\mathrm{Kg}$ ) da concentração residual dos isômeros do 
HCH no solo da área foco da Cidade dos Meninos, após o tratamento com cal, continua similar à observada antes do processo, como indicado por Oliveira (1994). Portanto, a tentativa de descontaminação efetuada na área, por meio da adição de cal, contrariamente ao laudo emitido pela empresa executora do tratamento, não foi eficaz para a remoção dos isômeros do HCH existentes no local.

Assim, é necessário determinar o uso futuro da área, para que seja realizado um tratamento, realmente eficaz, visando a eliminação ou remoção dos contaminantes a concentrações ambientais compatíveis às exigidas para o uso pré-estabelecido.

Após a constatação da ineficácia do tratamento executado na área, medidas emergenciais foram adotadas por parte das autoridades responsáveis pela mesma. Essas medidas incluíram instalação de cerca circundando a área foco tratada (aumentando a área em relação à originalmente cercada), instalação de sinalização advertindo sobre a presença de contaminantes no local e demolição das casas situadas nas proximidades da área foco (os moradores foram transferidos provisoriamente para outro local).

Com a adoção das medidas acima citadas, observou-se a diminuição da circulação de pessoas e a ausência de animais na área foco tratada. No entanto, ainda não foi determinada uma alternativa para a descontaminação desta.

É necessário ressaltar, para que as medidas adotadas diminuam o problema, que além da definição e implementação de metodologia adequada para a descontaminação da área, devem ser feitos, também, trabalhos de educação e conscientização junto à população.

A constatação de solo contaminado em quintais de residências e as plantações de culturas de subsistência e de frutas, cultivadas na área, representam uma rota importante de introdução de contaminantes, via cadeia alimentar, para a população. Portanto, medidas de controle de consumo destes alimentos por parte da população, devem ser adotadas para minimizar a contaminação humana.

\section{Referências}

BASTOS, L. H. P., 1999. Investigação da Contaminação do Solo por Organoclorados, na Cidade dos Meninos, em Duque de Caxias, Rio de Janeiro. Avaliação Dentro de um Novo Cenário, Após a Adição de Cal. Dissertação de Mestrado, Rio de Janeiro: Escola Nacional de Saúde Pública, Fundação Oswaldo Cruz.

BIJOS, G. M., 1961. Cinco anos entre os sanitaristas. Revista de Química e Farmácia, 6:13-79.

BRAGA, A. M. C. B., 1996. Contaminação Ambiental por Hexaclorociclohexano em Escolares na Cidade dos Meninos, Duque de Caxias, Rio de Janeiro. Dissertação de Mestrado, Rio de Janeiro: Escola Nacional de Saúde Pública, Fundação Oswaldo Cruz.

DIAS, A. E. X. O.; OLIVEIRA, R. M. \& BASTOS, L. H. P., 1997. Hexachlorocyclohexane $(\mathrm{HCH})$ case study: Cidade dos Meninos, Duque de Caxias, R. J., Brazil. In: International Workshop on Organic Micropollutants in the Environment, Proceedings, p. 23, Rio de Janeiro: Instituto de Biofísica Carlos Chagas Filho, Universidade Federal do Rio de Janeiro. 
EDWARDS, A. C., 1975. Persistent Pesticides in the Environment. Cleveland: CRC Press/Rothamsted Experimental Station.

EPA (Environmental Protection Agency), 1994. Method 8080A - Test Methods for Evaluating Solid Waste. Washington, DC: EPA.

FEEMA (Fundação Estadual de Engenharia e Meio Ambiente), 1991. BHC Abandonado na Cidade dos Meninos, Município de Duque de Caxias, R. J. Rio de Janeiro: FEEMA. (mimeo.)

IARC (International Agency for Research on Cancer), 2002. Summaries \& Evaluations - Hexachlorocyclohexanes - Group 2B. Lyon: IARC.

LIN, M. T. \& HSIEH, D. P. H., 1973. Lithium triethylborohydride. An exceptionally powerful nucleophile in displacement reactions with organic halides. Journal of the American Chemical Society, 95:1669-1671.

MELLO, J. L., 1999. Avaliação da Contaminação por HCH e DDT, dos Leites de Vaca e Humano, Provenientes da Cidade dos Meninos, Duque de Caxias - R. J. Dissertação de Mestrado, Rio de Janeiro: Escola Nacional de Saúde Pública, Fundação Oswaldo Cruz.
OLIVEIRA, R. M., 1994. Estudo da Contaminação do Solo e do Pasto Causada por Hexaclorociclohexanos (HCH) na Cidade dos Meninos em Duque de Caxias, R. J. Dissertação de Mestrado, Rio de Janeiro: Escola Nacional de Saúde Pública, Fundação Oswaldo Cruz.

PICÓ, Y.; MOLTÓ, C. J. \& FRONT, G., 1994. Solid phase techniques in the extraction of pesticides and related compounds from foods and soils. Journal of Microcolumn Separations, 6:331-359.

WHO (World Health Organization), 1991. Environmental Health Criteria 124: Lindane. Geneva: WHO.

WHO (World Health Organization), 1992. Environmental Health Criteria 123: Alpha- and BetaHexachlorocyclohexanes. Geneva: WHO.

Recebido em 6 de maio de 2002

Versão final reapresentada em 23 de agosto de 2002

Aprovado em 12 de novembro de 2002 